Phosphorus Research Bulletin Vol. 36 (2020) pp. 015-022

\title{
A COMPARITIVE IN VITRO BIOACTIVY EVALUATION OF POLYVINYLIDENE FLUORIDE AND POLYCAPROLACTONE INCORPORATED WITH AMORPHOUS CALCIUM PHOSPHATE PARTICLES
}

\author{
Hasnat Zamin ${ }^{1}$, Takeshi Yabutsuka ${ }^{*}$, Shigeomi Takai ${ }^{1}$, Hiroshi Sakaguchi ${ }^{2}$ \\ (*Corresponding author: yabutsuka@energy.kyoto-u.ac.jp) \\ ${ }^{1}$ Graduate School of Energy Science, Kyoto University, Kyoto 606-8501 Japan \\ ${ }^{2}$ Institute of Advanced Energy, Kyoto University, Uji, Kyoto 611-0011 Japan
}

Keywords: Polyvinylidene fluoride, Polycaprolactone, Bioactivity, Simulated body fluid, Apatite Nuclei, Apatite-forming ability.

A comparative study of polyvinylidene fluoride (PVDF) and polycaprolactone (PCL) incorporated with particles of amorphous calcium phosphate on the induction of apatite formation ability in simulated body fluid (SBF)-biomimetic environment has been investigated. PVDF and PCL films containing amorphous calcium phosphate particles were fabricated using the solvent casting method. These amorphous calcium phosphate particles were highly active in inducing apatite formation. Results showed that both PCL and PVDF successfully resulted in apatite formation in SBF. However, it was found that the amount of amorphous calcium phosphate particles and the induction period of the apatite formation in SBF significantly varied for PVDF and PCL. These types of bioactive polymeric composite materials are attractive candidates as bone restorative materials with flexibility as well as high apatite forming ability.

(Received Aug 28, 2020; Accepted Sep 11, 2020)

\section{INTRODUCTION}

Polymers, in general, are lightweight, relatively inexpensive, and have excellent mechanical toughness while showing biocompatibility and/or biodegradability. These properties make polymers highly suitable for application as biomaterials independently or in the form of multifunctional composites in combination with other organic or inorganic functional materials ${ }^{1}$.

Bone is a natural composite in which inorganic components are embedded in collagen fibrils. Bioactive ceramics are attractive candidates as bone implants, as they directly bond with bone by forming apatite layer compared to non-bioactive materials which get encapsulated with non-calcified organic tissue and is isolated from the surrounding bone tissue. This tissue formation is the immune defense response of the human body against the exogenous substances. However, ceramics are highly brittle, which makes it challenging to fit in the defect area and have higher young modulus, which can cause stress shielding effects after implantation. One of the most suitable alternates is polymer-bioceramic composites to mimic natural bone properties.

An effective approach to provide biological interaction between the non-bioactive implant and the host tissue is to coat or integrate the implants with suitable bioactive materials. Various bioactive materials such as bioactive glasses and calcium phosphates including hydroxyapatite can make the connection between the host bone and the implant which helps in tissue regeneration.

Biomimetic methods such as simulated body fluid (SBF) treatment is one of the suitable methods for the synthesis of calcium phosphate based bioactive materials with a similar composition to human bones ${ }^{2-4}$. SBF has inorganic ion concentration similar to that of human blood plasma and can reproduce spontaneous hydroxyapatite formation on the surface of bioactive materials. When either the $\mathrm{pH}$, temperature or the concentration of SBF is raised, fine particles of calcium phosphate are precipitated in the fluid in the absence of the bioactive ceramics. In previous studies, based on this mechanism amorphous calcium phosphate particles were fabricated from the SBF by raising its concentration, $\mathrm{pH}$ and temperature and it was found that these particles actively induce hydroxyapatite formation in conventional SBF, and hence were named as apatite nuclei $(\mathrm{AN})^{5,6}$. Various bioactive polymers, ceramic, and metals have been successfully prepared through biomimetic apatite nuclei treatment ${ }^{7-10}$.

Polyvinylidene fluoride (PVDF) and polycaprolactone (PCL) are semicrystalline biocompatible polymers. PVDF is a non-biodegradable fluoropolymer known for its electroactive properties ${ }^{11}$. Piezoelectric materials 
display significant potential for tissue engineering and regeneration. Piezoelectric materials are widely being utilized for tissue repair applications, especially in bone repair, where charges induced in the materials by mechanical stress can enhance bone formation ${ }^{12}$. Ficat et al. showed the effect of implanted piezoelectric PVDF film on bone regeneration ${ }^{13}$. Being a piezoelectric polymer, also showing high elasticity and good processability, PVDF is highly suitable for biomedical applications such as bone implants, vascular grafts, neural regeneration, biosensing applications, etc. ${ }^{14,15}$.

Whereas PCL is a biodegradable aliphatic polyester and has good processability due to its low glass transition temperature $\left(\sim-60^{\circ} \mathrm{C}\right)$ and melting temperature $\left(\sim 60^{\circ} \mathrm{C}\right)$. PCL also has a low biosorption rate suitable enough for bone tissue regeneration, which makes it a good candidate for bone tissue engineering $^{16}$

However, both PVDF and PCL are bioinert, which means that they lack direct bone-bonding ability. In this work, a comparative study has been done to impart bioactivity and discuss the apatite forming ability of PVDF and PCL incorporated with $\mathrm{AN}$ in the SBF environment.

\section{MATERIALS AND METHODS}

\section{Preparation of $A N$}

Firstly, 2.0SBF, having twice the concentration of inorganic ions compared to the normal SBF, was prepared. Then, the $\mathrm{pH}$ was raised to 8.2 at $36.5^{\circ} \mathrm{C}$ using tris(hydroxymethyl)aminomethane (THAM; Hayashi Pure Chemical Ind., Ltd., Osaka, Japan), and the solution was kept at the same temperature for 1 day. After 1 day, fine particles precipitated in the solution, which were filtered by vacuum filtration using a filter paper having $50 \mathrm{~nm}$ for average pore size (Merck Millipore, USA). Finally, these particles were characterized using field emission scanning electron microscopy (SEM; SU6600, Hitachi High-Technologies Corporation, Tokyo, Japan), energy dispersive X-ray spectrometry (EDS; XFlash ${ }^{\circledR}$ 5010, Bruker, Germany), X-ray diffraction (Ultima IV, Rigaku, Japan), and Fourier transform infrared spectroscopy (FT-IR; FT-720, Horiba, Ltd., Kyoto, Japan).

\section{Fabrication of composite films}

The solvent casting technique was used for the fabrication of films. PVDF (Sigma-Aldrich Japan, Tokyo, Japan) was dissolved in dimethylformamide (DMF) (Fujifilm Wako Pure Chemical Corporation, Osaka, Japan). An appropriate amount of AN was added to the solution to maintain a weight percentage of 0 wt. $\%, 10$ wt. $\%, 20$ wt. $\%$, and 30 wt. $\%$ of particles in PVDF. Magnetic stirring and subsequent ultrasonication were performed to disperse the AN in the PVDF solution uniformly. Then this solution was deposited as film onto the aluminum substrate using doctor blade method. Finally, the deposited films were kept at $90^{\circ} \mathrm{C}$ for 4 hours for drying, which were peeled off from the substrate after drying.

A similar method was used to fabricate PCL films. Acetone (Fujifilm Wako Pure Chemical) was used to dissolve PCL (Fujifilm Wako Pure Chemical), and an appropriate amount of AN were added to the solution to maintain a weight percentage of $0 \mathrm{wt} . \%$, 10 wt.\%, 20 wt.\%, and 30 wt.\% of AN in PCL. Similarly, magnetic stirring, ultrasonication, and doctor blade deposition of films on the aluminum substrate were performed. Instead of drying at $90^{\circ} \mathrm{C}$, the deposited films were kept at room temperature for the evaporation of the acetone and peeled off after drying.

The surface of the films was observed by (SEM; SU6600, Hitachi High-Technologies Corporation, Tokyo, Japan), energy dispersive X-ray spectrometry (EDS; XFlash ${ }^{\circledR}$ 5010, Bruker, Germany), thin-film X-ray diffraction (XRD; Rint 2500, Rigaku Corporation, Tokyo, Japan). Prior to SEM and EDX observation, the samples were coated with Au using the sputtering method. For SEM and EDX analysis acceleration voltage of $20 \mathrm{kV}$, an emission current of $32 \mu \mathrm{A}$ was used. XRD measurement of samples were performed at an angle step of $1^{\circ} \mathrm{min}^{-1}$. In order to compare the difference of hydrophilicity, the water contact angle of PVDF and PCL films was measured by contact angle meter (CAX-150, Kyowa Interface Science, Saitama, Japan). The surface roughness was measured by ultra-precision point autofocus probe 3D measuring instrument (NH-3SP, Mitaka Kohki Co., Ltd., Tokyo, Japan) to evaluate the difference of the roughness between PVDF and PCL films.
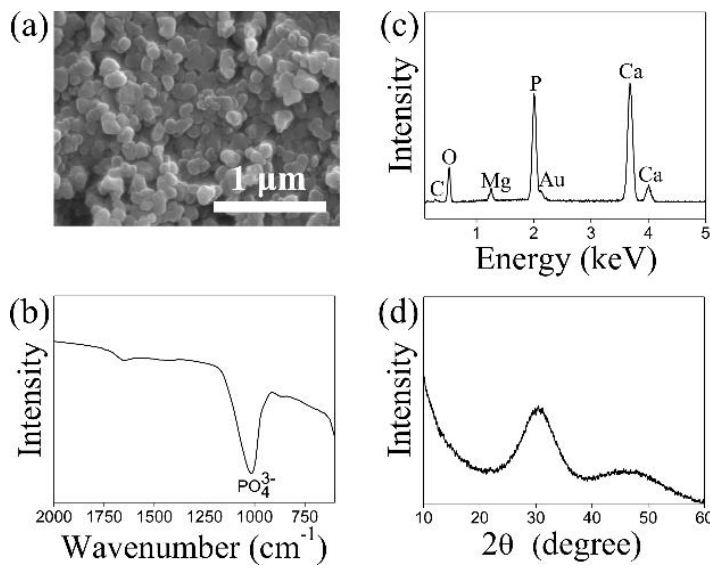

FIGURE 1 (a) SEM image, (b) FTIR plot, (c) EDX profile and (d) XRD plot of AN 


\section{Evaluation of bioactivity}

The prepared films were immersed in SBF solution for 1 day and 3 days to test in vitro bioactivity ${ }^{17}$. The surface of the films was observed by SEM, EDX, and thin-film XRD to evaluate apatite forming ability.

\section{RESULTS AND DISCUSSION.}

Figure 1 shows the SEM image, EDX profile, FTIR, and XRD plot of the AN. Spherical particles having a size around 100-200 $\mathrm{nm}$ were observed in the SEM image. The FTIR plot showed $\mathrm{P}=\mathrm{O}$ stretching from the peak around $1000 \mathrm{~cm}^{-1}$, and the presence of a peak around $1650 \mathrm{~cm}^{-1}$ might be due to the adsorbed atmospheric $\mathrm{CO}_{2}$. $\mathrm{Ca}$ and $\mathrm{P}$ peaks were detected in the EDX elemental analysis. Mg peak was also detected in the EDX, implying that $\mathrm{Mg}$ was incorporated in the AN. While the XRD shows a broad hump around $31^{\circ}$ instead of strong peaks as in

(a)

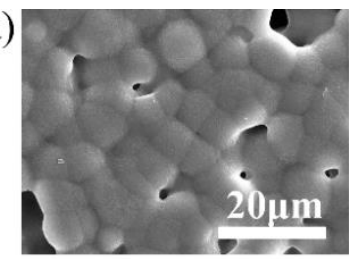

(b)

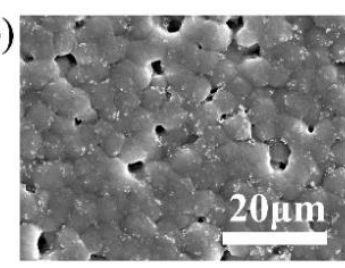

(c)

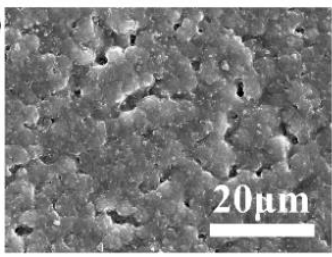

(d)

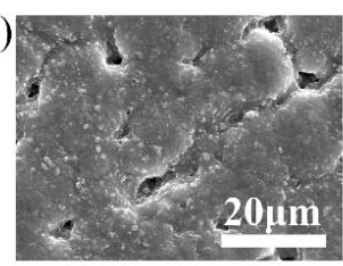

(e)

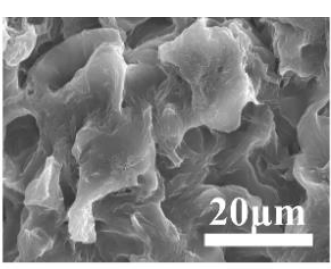

(f)

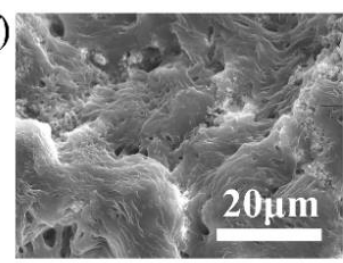

(g)

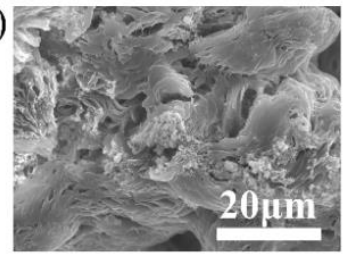

(h)

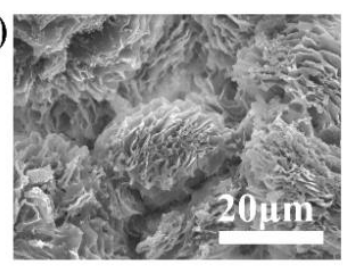

the case of hydroxyapatite. These results indicate that these were fine-sized amorphous calcium phosphate particles.

Figure 2 shows SEM images and EDX profiles comparing properties of the surfaces of the $0 \mathrm{wt} . \%$, 10 wt. $\%, 20$ wt. $\%$ and 30 wt. $\%$ AN-PVDF and AN-PCL films. For PVDF, the SEM images revealed globular surface morphology, and $\mathrm{C}$ and $\mathrm{F}$ peaks were detected in the EDX. In the case of PCL, the SEM images revealed highly rough surface morphology, and $\mathrm{C}$ and $\mathrm{O}$ peaks were obtained in the EDX profiles. As it can be noticed in the SEM images that the AN were exposed on the surface of the matrix. In the EDX profiles, $\mathrm{Ca}$ and $\mathrm{P}$ peaks are obtained from AN, which increased as the content of AN increased in PVDF and PCL. Similarly, Mg peak is obtained from AN for films having higher wt.\% of $\mathrm{AN}$ in PVDF/PCL, i.e. $20 \& 30$ wt.\% AN-PVDF/PCL films.

Figure 3 compares the surface roughness of 0 wt.\% apatite nuclei-polyvinylidene fluoride (AN-PVDF) and 0 wt.\% apatite nuclei-
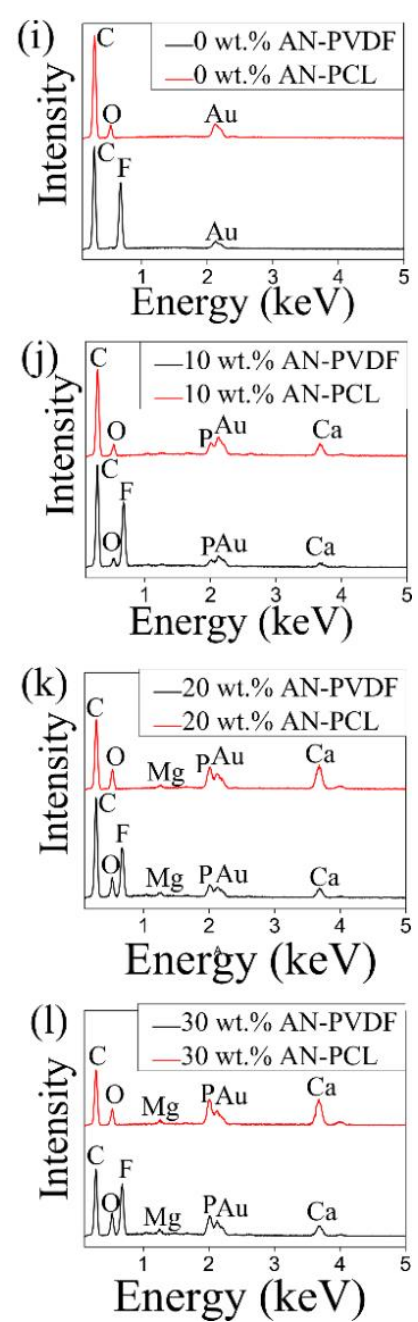

FIGURE 2 (a-h) SEM images of the surface of (a) 0 wt. $\%$, (b) 10 wt.\%, (c) 20 wt. $\%$ and (d) 30 wt. $\%$ AN-PVDF and (e) 0 wt. $\%$ (f) $10 \mathrm{wt} . \%$, (g) $20 \mathrm{wt} . \%$ and (h) $30 \mathrm{wt} . \%$ AN-PCL films. (i-l) EDX profiles comparing properties of (i) 0 wt.\% (j) 10 wt. $\%$, (k) 20 wt.\% and (l) 30 wt.\% AN-PVDF and AN-PCL films. 
(a)

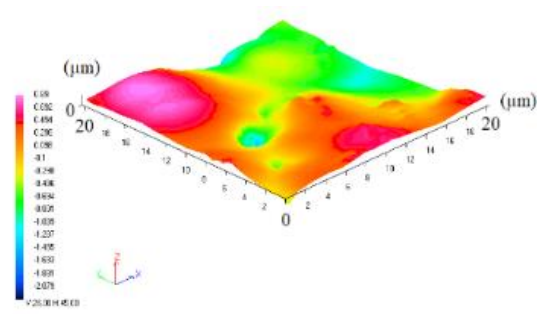

(b)

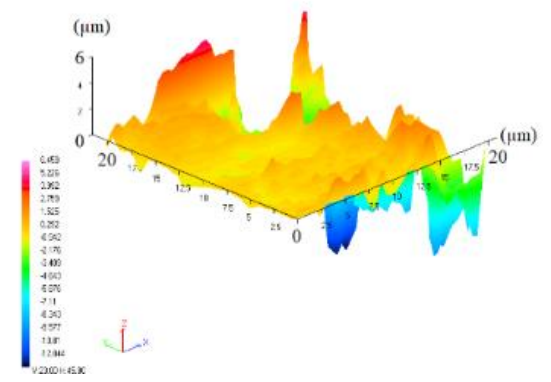

FIGURE 3 3D images of surface of (a) 0 wt.\% AN-PVDF and (b) 0 wt. $\%$ AN-PCL films.

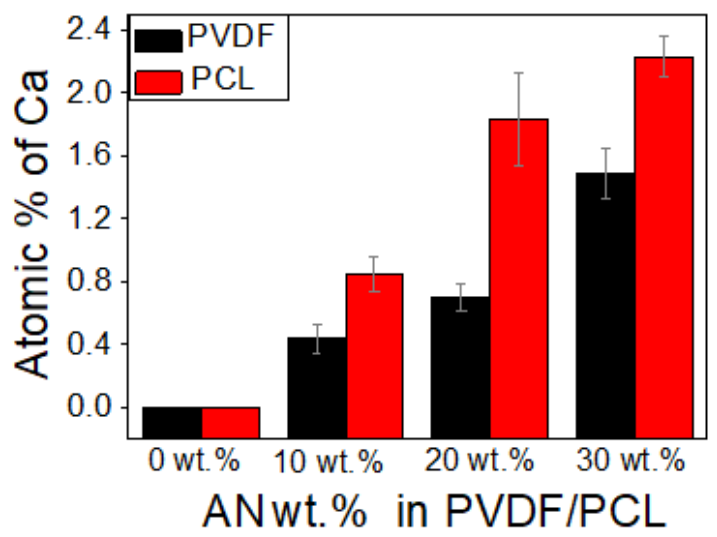

FIGURE 4 Comparison of atomic $\%$ of $\mathrm{Ca}$ on the surface of 0 wt. $\%, 10$ wt. $\%, 20$ wt. $\%$ and 30 wt. $\%$ AN-PVDF and AN-PCL films w.r.t. C, O, P \& F.

polycaprolactone (AN-PCL) films. As it can be observed from the $3 \mathrm{D}$ images, the surface was highly roughened for AN-PCL film compared to AN-PVDF film. The $\mathrm{R}_{\mathrm{a}}$ (average roughness) values were found to be $\sim 0.24$ and $\sim 1.37 \mu \mathrm{m}$ for the $0 \mathrm{wt} . \%$ AN-PVDF and 0 wt. $\%$ AN-PCL film, respectively.

Figure 4 shows the EDX surface elemental scanning to measure atomic percentage of $\mathrm{Ca}$ with respect to other elements $(\mathrm{C}, \mathrm{O}, \mathrm{P} \& \mathrm{~F})$ exposed on the surface of the films. The error bar represents the standard deviation from three readings obtained for each type of sample. It can be observed that the atomic \% of $\mathrm{Ca}$ increases with increasing the wt.\% of AN in PVDF/PCL. Furthermore, for each wt.\% of $\mathrm{AN}$ in PVDF/PCL, higher atomic\% of $\mathrm{Ca}$ was observed for PCL films compared to the PVDF films suggesting higher exposure of $\mathrm{AN}$ in PCL due to higher surface roughness.

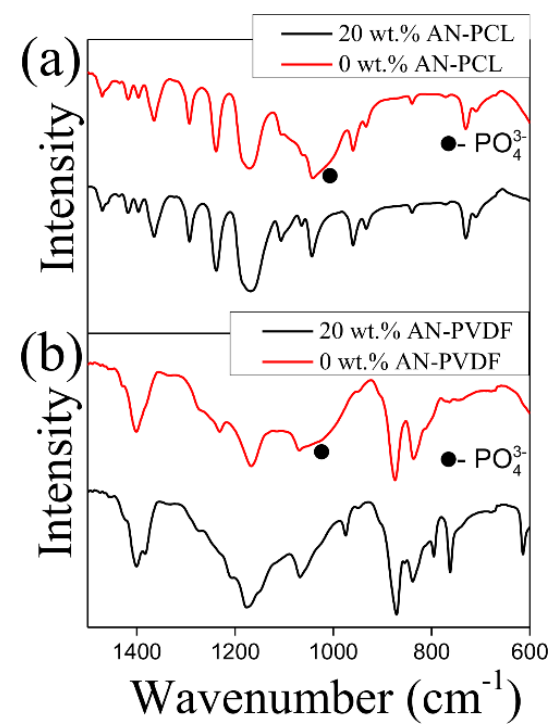

FIGURE 5 FTIR plots comparing 0 wt.\% and 20 wt.\%, (a) AN-PVDF and (b) AN-PCL films.

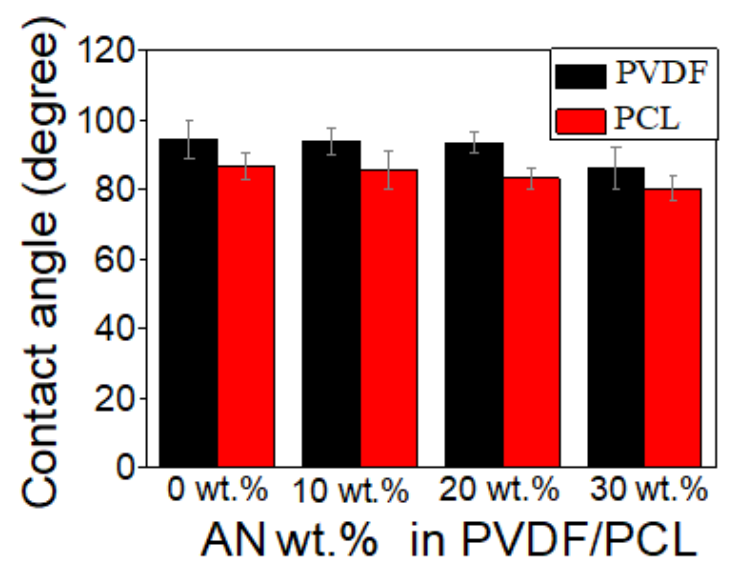

FIGURE 6 Comparison of water contact angle on $0 \mathrm{wt} \%$, 10 wt. $\%, 20$ wt.\% and 30 wt.\% AN-PVDF and AN-PCL films.

Figure 5 compares the FTIR spectra only for 0 wt.\% and 20 wt.\% AN-PVDF and AN-PCL films for brevity. A hump-shaped peak around $1000 \mathrm{~cm}^{-1}$ is obtained from $\mathrm{PO}_{4}{ }^{3-}$ instead of a distinct peak, possibly due to the overlap of the peak of AN with PVDF/PCL. This further corroborates EDX results suggesting that AN were incorporated inside the films.

Figure 6 shows the water contact angle of $0 \mathrm{wt} . \%$, 10 wt. $\%, 20$ wt. $\%$ and 30 wt. $\%$ AN-PVDF and AN-PCL films. The error bar represents the standard deviation from three readings obtained for each type of sample. The average water contact angle for pure PVDF film was found to be $94.6^{\circ}$, and for pure PCL film was found to be $86.7^{\circ}$. No significant changes in contact angle were observed at intermediate weight percentages of AN in PVDF but a higher weight percentage of $30 \mathrm{wt} \% \mathrm{AN}$ in PVDF resulted in a 
(a)

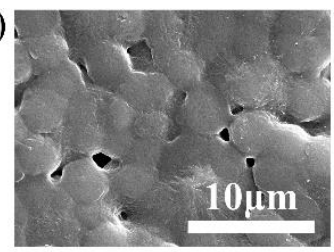

(b)

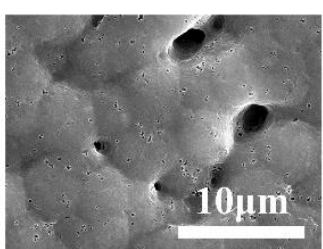

(c)

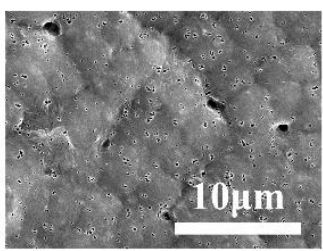

(d)

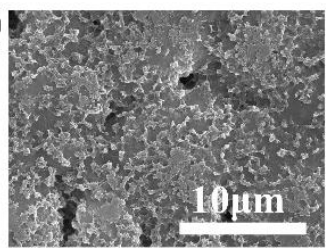

(e)

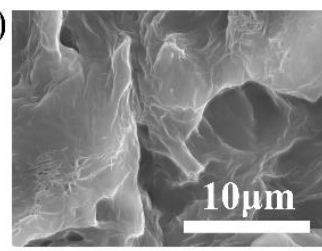

(f)

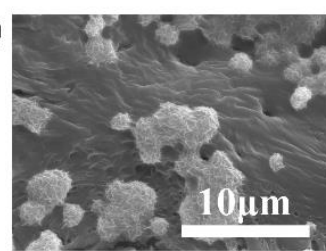

(g)

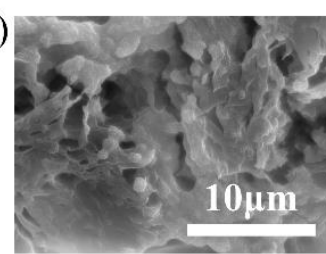

(h)

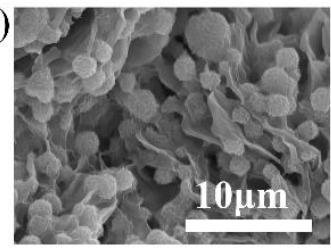

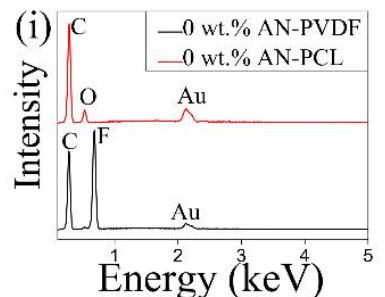

(j) $-10 \mathrm{wt.} \%$ AN-PVDF

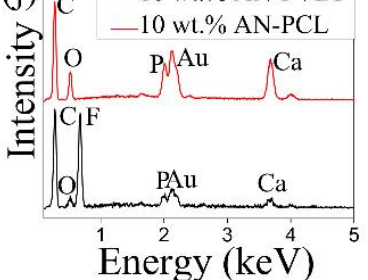

(k) $-20 \mathrm{wt} . \%$ AN-PVDF

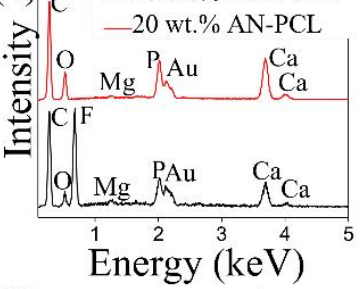

(1) -30 wt.\% AN-PVDF

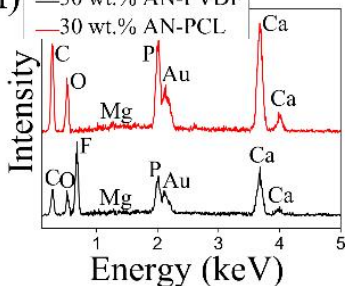

FIGURE 7 (a-h) SEM images of the surface of (a) 0 wt.\%, (b) $10 \mathrm{wt} . \%$, (c) $20 \mathrm{wt} . \%$ and (d) $30 \mathrm{wt} . \%$ AN-PVDF and (e) 0 wt.\% (f) 10 wt.\%, (g) 20 wt.\% and (h) 30 wt.\% AN-PCL films after 1 day SBF immersion. (i-l) EDX profiles comparing properties of (i) 0 wt.\% (j) $10 \mathrm{wt} . \%$, (k) $20 \mathrm{wt} . \%$ and (1) $30 \mathrm{wt} . \%$ AN-PVDF and AN-PCL films after 1 day SBF immersion.

slight decrease of the contact angle. While in the case of AN- PCL films, the decrease in contact angle was also not significant, which gradually decreased with the increase of AN concentration in PCL.

Figure 7 shows SEM images and EDX profiles comparing properties of the surface of the $0 \mathrm{wt} . \%, 10$ wt.\%, 20 wt. $\%$ and 30 wt.\% AN PVDF and AN-PCL films after 1 day SBF immersion. No changes in the surface morphology were observed for AN-PVDF films except for $30 \mathrm{wt} \%$ AN-PVDF film, where partially formed precipitates were observed. The EDX plots also showed a similar intensity of the $\mathrm{Ca}$ and $\mathrm{P}$ peaks compared prior to the SBF treatment for the films with no precipitates formed. Minute pores were formed on the surface of these films, presumably due to the dissolution of AN in SBF. In the case of AN-PCL films, hemispherical precipitates were observed on $10 \mathrm{wt} . \%, 20 \mathrm{wt} . \%$ and $30 \mathrm{wt} . \%$ films, and relative higher intensity $\mathrm{Ca}$ and $\mathrm{P}$ peaks were observed for 20 wt. $\%$ and 30 wt. $\%$ films compared to films prior to SBF treatment. However, it is noticeable from the SEM images and high-intensity $\mathrm{C}$ peak in the EDX profiles that these precipitates partially covered the surface, and the surface coverage increased with the increase in $\mathrm{AN}$ in content in PCL.

Figure 8 SEM images and EDX profiles comparing properties of the surfaces of the $0 \mathrm{wt} . \%$, 10 wt. $\%, 20$ wt. $\%$ and 30 wt. $\%$ AN-PVDF and AN-PCL films after 3 days SBF immersion. Partially formed crystallite on the surface of 30 wt. $\%$ AN-PVDF film, which was observed for 1 day SBF immersion, had fully developed into flakes like crystallite representing bone-like apatite. Low-intensity $\mathrm{F}$ peaks and high $\mathrm{Ca}$ and $\mathrm{P}$ peaks observed in the EDX indicates that the surface was fully covered by these crystallites. While no changes on the surface morphology were observed on $0 \mathrm{wt} . \%$ 10 wt. $\%$ and 20 wt. $\%$ AN-PVDF films. Similarly, in the case of AN-PCL films, hemispherical precipitates formed after 1 day SBF immersion had 
(a)

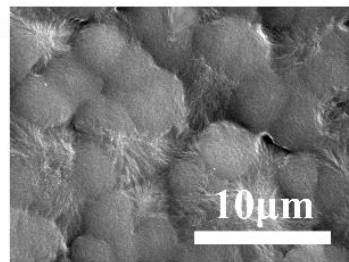

(b)

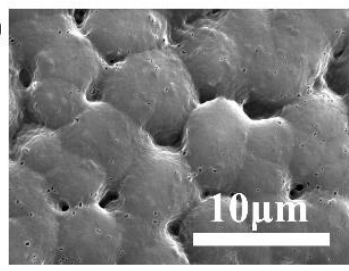

(c)

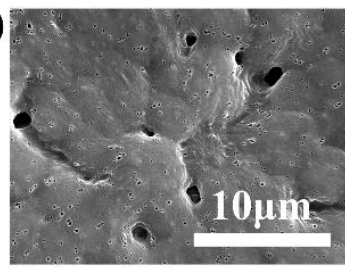

(d)

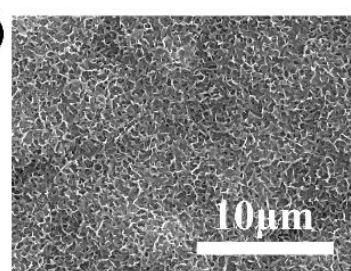

(e)

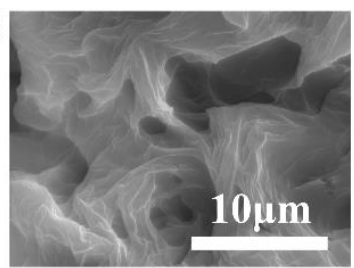

(f)

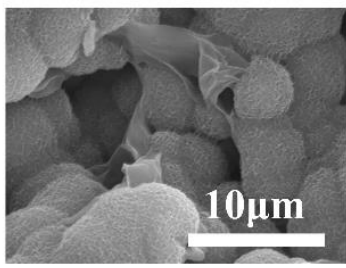

(g)

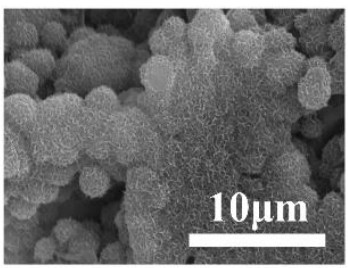

(h)

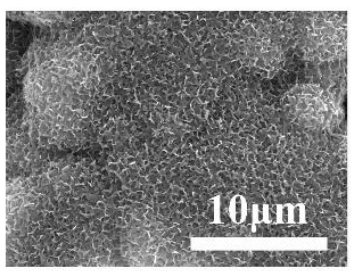

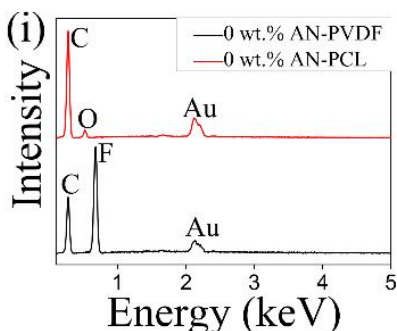
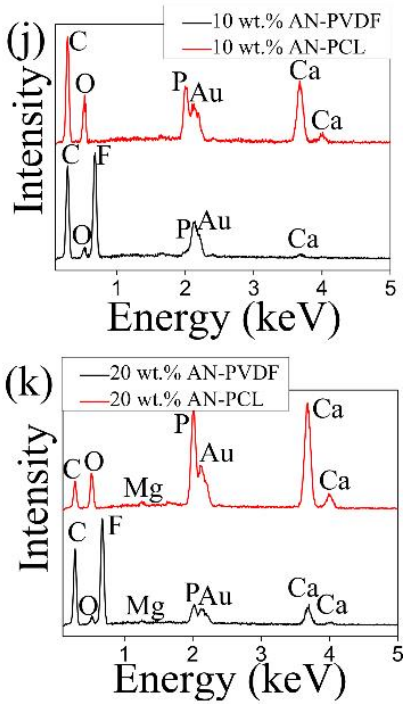

(1) $-30 \mathrm{wt. \%}$ AN-PVDF

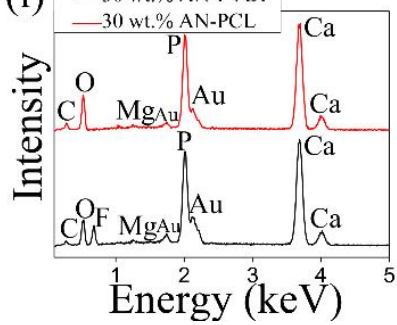

FIGURE 8 (a-h) SEM images of the surface of (a) $0 \mathrm{wt} \%$, (b) $10 \mathrm{wt} . \%$, (c) $20 \mathrm{wt} \%$ and (d) $30 \mathrm{wt} . \%$ AN-PVDF and (e) $0 \mathrm{wt} \%$ (f) $10 \mathrm{wt} \%$, (g) $20 \mathrm{wt} . \%$ and (h) $30 \mathrm{wt} . \%$ AN-PCL films after 3 day SBF immersion. (i-l) EDX profiles comparing properties of (i) $0 \mathrm{wt} . \%$ (j) $10 \mathrm{wt} \%$, (k) $20 \mathrm{wt} . \%$ and (l) $30 \mathrm{wt} . \%$ AN-PVDF and AN-PCL films after 3 day SBF immersion.
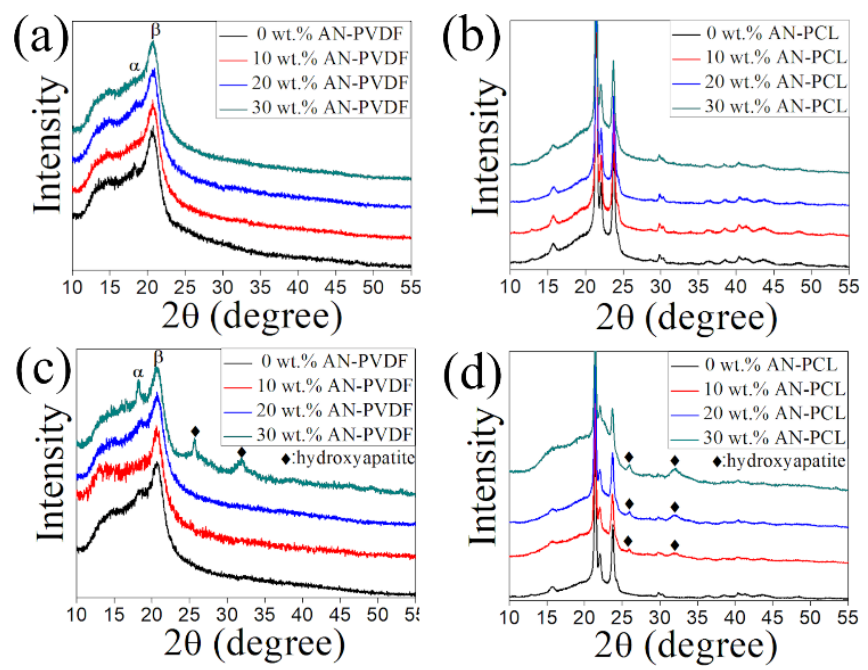

FIGURE 9 XRD plots of (a, c) AN-PVDF and (b, d) AN-PCL films after (a, b) 1 day and (c, d) 3 days SBF immersion. 
fully developed into flakes like crystallites representing bone-like apatite for $10 \mathrm{wt} \%$, $20 \mathrm{wt} \%$, and $30 \mathrm{wt} \%$ AN-PCL films. In the EDX result for 20 wt.\% and 30 wt.\% AN-PCL films low intensity of C peak and high intensity $\mathrm{Ca}$ and $\mathrm{P}$ peaks were observed, suggesting high surface coverage by the crystallites, which increased with increasing the content of AN in PCL.

Figure 9 compares the XRD plots of 0 wt. $\%, 10$ wt. $\%, 20$ wt. $\%$ and 30 wt.\% AN-PVDF and AN-PCL films after 1 day and 3 days immersion in SBF. PVDF shows five distinct crystalline phases based on the chain conformations, which are $\alpha, \beta, \gamma, \delta$, and $\varepsilon$. As observed from the XRD, the AN-PVDF films mainly consist of $\alpha$ and $\beta$ phase, represented by peaks at $18.3^{\circ}$ and $20.6^{\circ}$, respectively. The $\beta$ phase is a highly piezoelectric phase of PVDF, which was observed for all AN-PVDF films. Nucleating fillers and solvent casting technique have been reported to cause $\beta$ phase dominance in $\mathrm{PVDF}^{18}$. Peaks representing hydroxyapatite at $25.8^{\circ}$ and $31.7^{\circ}$ were observed for 30 wt.\% AN-PVDF film after 3 days SBF immersion. Similarly, in the case of $10 \mathrm{wt} \%, 20$ wt.\% and 30 wt.\% AN-PCL films, peaks representing hydroxyapatite were detected in XRD after 3 days immersion in SBF. The intensity of these peaks increased with the increase of the content of AN in PCL. Though the precipitates were deposited on the surface of these films after 1 day SBF immersion, no peaks of hydroxyapatite were detected after 1 day SBF immersion, indicating that the crystallinity or the surface coverage of the films was not enough to be detected in the XRD. These XRD results complement the SEM and the EDX result.

The formation of hydroxyapatite from the constituent ions in an aqueous solution is represented by the following chemical equilibrium.

$$
10 \mathrm{Ca}^{2+}+6 \mathrm{PO}_{4}{ }^{3-}+2 \mathrm{OH}^{-} \rightleftharpoons \mathrm{Ca}_{10}\left(\mathrm{PO}_{4}\right)_{6}(\mathrm{OH})_{2}
$$

The ionic activity product (IP) of the apatite in the solution is depicted by the following formula where ' [] ' is the concentration of each ion and ' $\gamma$ ' is the activity coefficient.

$$
\begin{aligned}
\mathrm{IP} & \rightleftharpoons\left(\gamma \mathrm{Ca}^{2+}\right)^{10}\left(\gamma \mathrm{PO}_{4}{ }^{3-}\right)^{6}\left(\gamma \mathrm{OH}^{-}\right)^{2} \\
& \times\left[\mathrm{Ca}^{2+}\right]^{10}\left[\mathrm{PO}_{4}{ }^{3-}\right]^{6}\left[\mathrm{OH}^{-}\right]^{2}
\end{aligned}
$$

The conventional SBF at physiological condition, which is pH 7.40 at $36.5^{\circ} \mathrm{C}$, is supersaturated against hydroxyapatite. However, generally because of high energetic obstacles toward the hydroxyapatite formation in SBF, it is induced only on the surface of specific materials, as in the case of bioactive materials. The increase in the concentration of $\mathrm{Ca}^{2+}$ ions would increase the ionic product and degree of supersaturation, which is $\mathrm{IP} / K_{\mathrm{s}} \quad\left(K_{\mathrm{s}}=\right.$ solubility product), thus favoring hydroxyapatite nucleation ${ }^{19}$. In the light of previous studies, it was found that AN were amorphous calcium phosphate, which is easier to dissolve in SBF compared to the crystalline hydroxyapatite. It is considered that this leads to an increase in the concentration of calcium ions, accelerating hydroxyapatite nucleation on the substrate ${ }^{7-10}$.

In this study, both PVDF and PCL successfully formed hydroxyapatite layer in SBF. However, PCL, required a lower concentration of $\mathrm{AN}$ and shorter induction time of hydroxyapatite formation in SBF compared to PVDF. The PCL films, as observed from the SEM analyses, showed deposited hemispherical precipitates in 1 day immersion period for all concentrations of AN in PCL while no hydroxyapatite formation was observed for the films less than 30 wt.\% of AN in PVDF. To clarify this difference in hydroxyapatite forming ability, water contact measurements and surface roughness analyses of the films were performed as depicted in Figures 3 and 6. Surface wettability determines the interaction between the SBF and the substrate, thus effecting the apatite formation. Though at a higher weight percentage of $30 \mathrm{wt} . \%$ of AN in PVDF resulted in a slight decrease of water contact angle, a large difference in the contact angle between PVDF and PCL films was not observed. This result indicates that in this case, the wettability factor might only have contributed a little in hydroxyapatite formation. It is considered that the difference in the hydroxyapatite formation is because of the higher surface roughness of the PCL films compared to the PVDF films, which resulted in exposing a higher amount of $\mathrm{AN}$ to the SBF in the PCL films.

\section{CONCLUSION}

PVDF and PCL successfully showed apatite forming ability in SBF by incorporation of AN. It was found that for all compositions of $\mathrm{AN}$ in PCL precipitates were deposited in 1 day immersion in $\mathrm{SBF}$, which fully developed into hydroxyapatite layer in 3 days SBF immersion. Whereas PVDF formed hydroxyapatite layer at a higher weight percentage of 30 wt.\% AN in PVDF. This difference could be attributed to the higher surface roughness of PCL, which exposed a higher amount of AN to SBF. Such types of bioactive polymeric composites such as PCL and PVDF can serve as a suitable candidate for bone tissue engineering.

\section{REFERENCES}

1. J. Jordan, K. I. Jacob, R. Tannenbaum, M.A. 
Sharaf, I. Jasiuk, Mater. Sci. Eng. C, 393, 1-11 (2005).

2. K. Hata, T. Kokubo, T. Nakamura, T. Yamamuro, J. Am. Ceram. Soc., 78, 1049 -1053 (1995).

3. Y. Abe, T. Kokubo, T. Yamamuro, J. Mater. Sci.: Mater. Med., 1, 233-238 (1990).

4. H.-M. Kim, K. Kishimoto, F. Miyaji, T. Kokubo, T. Yao, Y. Suetsugu, J. Tanaka, T. Nakamura, J. Biomed. Mater. Res., 46, 228-35 (1999).

5. T. Yao, M. Hibino, S. Yamaguchi and H. Okada, US Patent, 8,178,066 (2012), Japanese Patent 5, 261, 712 (2013).

6. T. Yao, M. Hibino, T. Yabutsuka, US Patent, 8,512,732 (2013), Japanese Patent 5, 252, 399 (2013).

7. T. Yabutsuka, H. Mizutani, S. Takai, T. Yao, Trans. Mat. Res. Soc. Japan, 43, 143-147 (2018).

8. T. Yabutsuka, R. Karashima, S. Takai, T. Yao, Materials, 11, 1334 (2018).

9. T. Yabutsuka, K. Fukushima, T. Hiruta, S. Takai, T. Yao, J. Biomed. Mater. Res. B. Appl. Biomater., 106, 2254-2265, (2018).

10. T. Yabutsuka, K. Fukushima, T. Hiruta, S. Takai, T.Yao, Mater. Sci. Eng. C, 81, 349-358 (2017).

11. H. Kawai, Jpn. J. Appl. Phys., 8, 975-976 (1969).

12. G.W. Hastings, F.A. Mahmud, J. Biomed. Eng., 10, 515-521 (1988).

13. J. Ficat, G. Escourron, M.J. Fauran, R. Durroux, P. Ficat, C. Lacabanne, F. Micheron, Ferroelectrics, 51, 121-128 (1983).

14. C. Ribeiro, V. Sencadas, D. M. Correia, S.L. Méndez, Coll. Surf. B, 136, 46-55 (2015).

15. A. H. Rajabi, M. Jaffe, T. L. Arinzeh, Acta Biomaterialia, 24, 12-23 (2015).

16. N. Bölgen, Y.Z. Menceloglu, K. Acatay, I. Vargel, E. Piskin, J. Biomater. Sci. Polym., 16, 1537-1555, (2005).

17. T. Kokubo, H, Takadama, Biomaterials, 27, 2907-2915 (2006).

18. P. Martins, A.C. Lopes, S. L. Mendez, Prog. Polym. Sci., 39, 683-706 (2014).

19. C. Ohtsuki, T. Kokubo, T. Yamamuro, $J$. Non-Cryst. Solids, 143, 84-92 (1992). 